\title{
Electroclinical characteristics of seizures arising from the posterior cingulate cortex and surgical outcomes based on stereoelectroencephalography (SEEG)
}

\section{Zhaofen Yan}

Capital Medical University Sanbo Brain Hospital https://orcid.org/0000-0002-4291-3938

Jing Wang

Capital Medical University Sanbo Brain Hospital

Qin Qin Deng

Capital Medical University Sanbo Brain Hospital

\section{Yujiao Yang}

Capital Medical University Sanbo Brain Hospital

\section{Xing Feng}

Capital Medical University Sanbo Brain Hospital

Jian Zhou

Capital Medical University Sanbo Brain Hospital

YuGuang Guan

Capital Medical University Sanbo Brain Hospital

\section{Feng Zhai}

Capital Medical University Sanbo Brain Hospital

Mengyang Wang ( $\triangle$ yanzf327@sina.com )

Capital Medical University Sanbo Brain Hospital

Guoming Luan ( $\square$ luangm3@163.com )

Capital Medical University Sanbo Brain Hospital

\section{Research article}

Keywords: PCE, SEEG, electroclinical characteristics, anatomical-electrical-clinical correlations, surgical outcome

Posted Date: April 10th, 2020

DOI: https://doi.org/10.21203/rs.3.rs-21165/v1 
License: (c) (i) This work is licensed under a Creative Commons Attribution 4.0 International License. Read Full License 


\section{Abstract}

Background Seizures arising from the posterior cingulate cortex are rare, and few studies have to characterized the clinical presentation of such seizures within the anatomic context of the limbic network. We aimed to characterize the electrophysiological properties and clinical features of seizures arising from the PCC and surgical outcomes based on data from stereoelectroencephalography (SEEG).

Methods The present retrospective study included 12 patients with medically intractable epilepsy, all diagnosed with posterior cingulate epilepsy via stereoelectroencephalography (SEEG) at Sanbo Hospital between 2014 and 2018. This retrospective analysis included clinical semiology, scalp EEG/SEEG findings and surgical outcomes of twelve intractable PCE patients.

Results Six of the twelve patients reported auras, including vestibular responses (3/6),blurred vision (2/6), and fear and déjà vu(1/6). Clinical semiology included dialeptic seizures(3/12),automotor seizures(4/12) and complex motor behaviours(5/12). The classifications of scalp EEG varied, including bilateral regional posterior temporal-occipital discharges in eight cases, regional anterior-middle temporal discharges in two cases, and no epileptic discharge in two cases.In five patients, ictal onset occurred mainly in the tempo-parietal-occipital area.In ten patients, the seizure onset zone by SEEG occurred in the posterior cingulate gyrus, which spread to the medial temporal, precuneus, inferior parietal lobe and long insular gyrus. However, for the two patients with posterior cingulate lesions, the seizure onset zone was the contralateral hippocampal head that spread to the posterior cingulate lesion. One patient was seizure free after posterior cingulate gyrus lesion resection, but another had $50 \%$ fewer seizures by using SEEG-guided radiofrequency thermocoagulation. Nine patients who underwent epilepsy surgery were seizure-free(Engel IA: 7/9;EngelIB: 2/9). One patient showed a 30\% reduction in seizures by using SEEG-guided radiofrequency thermocoagulation but not surgical resection.

Conclusions Our findings indicate that the clinical characteristics of posterior cingulate epilepsy vary among patients, and recognition of a posterior cingulate focus is challenging, especially with MRInegative epilepsies.Scalp EEG is of little use when attempting to localize posterior cingulate seizures.For patients with pharmacoresistant posterior cingulateepilepsy, favourable outcomes (Engel class I) could be observed by using SEEG. The SEEG findingsin PCE showed that the seizure onset zone and epileptogenic zone were a set of interconnected regional systems.

Clinical trial registration Restrospective case reports do NOT need registration of clinical trials.

\section{Background}

Posterior cingulate epilepsy(PCE) is a good example of seizure semiology that can be misleading because the seizure arises in anatomically deep and 'non-eloquent' areas. The anatomical location of the posterior cingulate gyrus makes neurophysiological studies with scalp electrodes difficult.The posterior cingulate gyrus is part of the limbic network and a component of the Papez circuit with extensive and complicated functional connectivity[1].Furthermore,the electroclinical findings with posterior cingulate 
epilepsysuggest temporal lobeepilepsy[2].In 2008, Koubeissi etal. [3]analysed the propagation of parietal cingulate seizures with secondary mesial temporal involvement. Other reports of posterior cingulate epilepsy did not validate the localization with favourable surgical outcomes[1].Enatsu et al.[4] described six of seven patients who had posterior cingulate ictal onset identified by SEEG evaluations and showed bilateral asymmetric tonic seizures, hypermotor seizures, and dialeptic and automotor seizures. Five patients who underwent epilepsy surgery achieved good seizure freedom.

Stereo-electroencephalography (SEEG) enables precise recordings from deep cortical structures, such as limbic and para-limbic structures and their associated epileptic networks[5]. In recent decades, epilepsy surgery in patients has demonstrated favourable outcomes in terms of seizure freedom and improved neuropsychological development.For those patients, the safety and utility of the SEEG technique has been wellestablished.

In the present study, we investigated the electrical clinical characteristics of epilepsy originating from the posterior cingulate cortex,as well as the surgical results in patients with drug-refractory focal PCE,which was identified by SEEG exploration.

\section{Methods}

\section{Patients}

We enrolled twelve consecutive patients who had been diagnosed with focal PCE at the Epilepsy Center of Sanbo Brain Hospital between 2014 and 2018. Nine patients were right-handed males, and three were right-handed females. The twelve patients fulfilled the following criteria: (i)had SEEG recordings covering the posterior cingulate cortex; (ii)the PCG was within the epileptogenic zone; and(iii)the extent of surgical excision or radiofrequency thermocoagulation was limited or mostly limited to within the posterior cingulate cortex.All included patients and caregivers provided written informed consent to participate and for publication.

All patients underwent a rigorous evaluation performed in three steps as previously described:

(i) Pre-surgical evaluation included detailed history, neurological examination, long-term video-EEG monitoring (VEEG), high-resolution MRI and SEEG. PET scans were performed in eleven patients. MEG and neuropsychologicaltesting were performed in nine patients. The clinical characteristics of the included patients are shown in Table 1.

(ii)For every patient, all types of seizures were recorded.SEEG exploration planning was performed based on noninvasive data, including the hypothetical epileptogeniczone(s) (Ez) and the lesion when it was present. The patient's head was fixed in a standard stereotactic frame, and depth electrode targeting and trajectory were determined using arobotic system (ROSA;Medtech,Montpellier,France). The electrodes consisted of $8,10,12$ and 16 cylindrical 2.0 mm-long platinum contacts with diameters of $0.80 \mathrm{~mm}$ and 
intervals of $1.5 \mathrm{~mm}$. Weco-registered postoperative CT with preoperative MRI to confirm the final location of the SEEG contacts and electrodes.

The VEEG and SEEG data wereindependently reviewed by two epileptologists. We classified the seizure semiology at the ictal stage based on the semiological seizure classification proposed by Lüders et al. [6].The seizure onset zone and propagations[7]were retrospectively analysed from SEEG data and monitoring reports.We paid particular attention to electroanatomical relationships between the posterior cingulate cortex and adjacent structures.

(iii)Tailored resection: A patient management conference was held for each individual after enough seizures had been recorded (at least three to five seizures) to discuss the results and implications of the SEEG study and to collectively decide on a plan for resection.Subsequent to thismeeting and approximately 8-12 weeks after removal of the SEEGelectrodes, patients underwent a standard craniotomy for tailored resection of the hypothetical EZ.

(『)Follow-up: Seizure outcomes were evaluated according to Engel’s classification[8],neurological examination, EEG, high-resolution MRI and neuropsychological testing.

Table 1 Demographics and clinical features of patients $(n=12)$

\begin{tabular}{|c|c|c|c|c|c|c|c|c|c|c|}
\hline Patient & $\begin{array}{l}\text { Age/Se } \\
x \\
(\mathrm{M} / \mathrm{F})\end{array}$ & $\begin{array}{l}\text { Age at } \\
\text { Onset( }(Y)\end{array}$ & MRI & PET & MEG & $\begin{array}{l}\text { WAIS- } \\
\text { IV(FIQ/MQ) }\end{array}$ & Surgery & $\begin{array}{l}\text { Patholo } \\
\text { gy }\end{array}$ & $\begin{array}{l}\text { follow-up, } \\
\text { (month) }\end{array}$ & $\begin{array}{l}\text { Engel's } \\
\text { classification }\end{array}$ \\
\hline 1 & $17 / \mathrm{M}$ & 15 & - & PCG & & No & $\begin{array}{l}\text { Lt.RSC and PHG } \\
\text { RT-FC }\end{array}$ & & 20 & IIII \\
\hline 2 & $25 / \mathrm{F}$ & 14 & - & - & & $\begin{array}{l}\text { Pre:FIQ/MQ:85/98; } \\
\text { Post:FIQ/MQ:87/98 }\end{array}$ & $\begin{array}{ll}\text { Lt.PCG,RSC } & \text { RT- } \\
\text { FC } & \end{array}$ & - & 26 & $\mathrm{IB}$ \\
\hline 3 & $35 / \mathrm{F}$ & 16 & PCG & $\begin{array}{l}\text { LtP and } \\
\text { PCG }\end{array}$ & - & Pre:FIQ/MQ:110/110 & $\begin{array}{l}\text { Lt.PCG and RSC } \\
\text { RT-FC }\end{array}$ & - & 16 & III \\
\hline 4 & $22 / \mathrm{M}$ & 18 & Rt.PCG & Rt.TPO & Rt.T & Pre:FIQ/MQ:90/90 & Rt.PCG,RSC,Prc & FCDIIIB & 36 & Ia \\
\hline 5 & $13 / \mathrm{M}$ & 10 & Rt.P-O & No & NO & Pre:FIQ/MQ:90/80 & Rt.PCG,Prc,Cu & $\begin{array}{l}\text { brain } \\
\text { injury }\end{array}$ & 44 & Ia \\
\hline 6 & $12 / \mathrm{M}$ & 0.2 & Bil.TPO & Rt.TPO & No & No & Rt.Prc,Cu,PCG & FCDIC & 66 & Ia \\
\hline 7 & $18 / \mathrm{M}$ & 5 & - & - & NO & Pre:FIQ/MQ:60/54 & Lt.PCG,Cu & FCDIc & 34 & Ia \\
\hline 8 & $7 / \mathrm{M}$ & 6 & $\begin{array}{l}\text { Lt.thalamic } \\
\text { infarction }\end{array}$ & Lt.F and $T$ & No & Pre:FIQ/MQ:72/45 & $\begin{array}{l}\text { Lt.PCG,Lt.Prc RT- } \\
\text { FC }\end{array}$ & - & 12 & Ia \\
\hline 9 & $16 / \mathrm{M}$ & 13 & Lt.PCG & Lt.T & Lt.T & Pre:FIQ/MQ:119/128 & Lt.RSC RT-FC & - & 6 & Ia \\
\hline 10 & $24 / \mathrm{F}$ & 10 & Rt.ATL & Rt.TP & $\begin{array}{ll}\text { Rt.FT } \\
\end{array}$ & Pre:FIQ/MQ:101/96 & $\begin{array}{ll}\text { Rt.PCG } \\
\end{array}$ & FCD I c & 48 & Ia \\
\hline 11 & $18 / \mathrm{F}$ & 7 & Rt.ATL & Lt.P and Rt.T & Lt.P & Pre:FIQ/MQ:78/75 & Lt.PCG,RSC PCu & FCDIb & 41 & Ic \\
\hline 12 & $31 / \mathrm{M}$ & 3.5 & & $\begin{array}{l}\text { Lt.TP } \\
\end{array}$ & Lt.TO & No & Lt.PCG,RSC,Cu & FCD I c & 34 & Ia \\
\hline
\end{tabular}

\section{Results}

The main clinical data are detailed in Tables 1 and 2. 
Table 2 Features of scalp EEG,semiology and SEEG in patients $(n=12)$

\begin{tabular}{|c|c|c|c|c|c|}
\hline Patient & $\begin{array}{l}\text { interictal } \\
\text { Scalp EEG }\end{array}$ & aura & ictal Scalp EEG & ictal onset zone & early spread \\
\hline 1 & Bio-TPO & $\begin{array}{l}\text { Blurred } \\
\text { vision,dizzness }\end{array}$ & $\begin{array}{l}\text { Dialeptic } \rightarrow \quad \text { (olimentary } \quad \text { Bio-TPO } \\
+ \text { +Bil hands) }\end{array}$ & Lt.RSC,PHG & Lt.POS,HH \\
\hline 2 & Bil-TPO & Fear-déjà vu & $\begin{array}{llr}\begin{array}{l}\text { Vocalization } \\
\text { seizure } \rightarrow\end{array} & \begin{array}{r}\text { versive } \\
\text { seil-TPO } \\
\text { seizure } \rightarrow \text { GTCS }\end{array} & \end{array}$ & Lt.PCG,RSC & Lt.POJ,IPL \\
\hline 3 & - & vertigo & Dialeptic-tonic & Rt.HH & $\begin{array}{l}\text { Lt.HH,Lt.PCG,Lt. } \\
\text { RSC }\end{array}$ \\
\hline 4 & Bil.T & $\begin{array}{l}\text { Blurred } \\
\text { vision,dizzness }\end{array}$ & $\begin{array}{l}\text { Automotor(olimentary and Rt.F and T } \\
\text { Bil.Hand) }\end{array}$ & Lt.HH & Rt.PCG \\
\hline 5 & Rt.TPO & - & $\begin{array}{l}\text { Dialeptic } \rightarrow \text { Automotor(oli Rt. hemisphere } \\
\text { mentary and Rt.Hand) }\end{array}$ & Rt.RSC & Rt.HH,PCG,Prc \\
\hline 6 & Bil.TPO & - & $\begin{array}{l}\text { Dialeptic } \rightarrow \text { Automotor(oli diffuse } \\
\text { mentary and Rt.Hand }\end{array}$ & Rt.RSC,POS,Prc & Rt.PCG,TTG \\
\hline 7 & - & - & $\begin{array}{l}\text { Dialeptic } \rightarrow \text { Automotor(oli Bil.TPO } \\
\text { mentary and Bil.Hand) }\end{array}$ & Lt.RSC & Lt.HH,A,PHG \\
\hline 8 & Lt.hemisphere & & Dialeptic $\rightarrow$ complexmotor $\quad$ Lt.hemisohere & Lt.RSC,PCC,POS & $\begin{array}{l}\text { Lt.Prc,mr } \\
\text { (marginal ramus) }\end{array}$ \\
\hline 9 & Lt.FTP & - & Dialeptic $\rightarrow$ complexmotor Lt.FT & Lt.RSC & Lt.HH \\
\hline 10 & Rt.T & $\begin{array}{l}\text { Blurred } \\
\text { vision,dizzness,au } \\
\text { ditory,fear }\end{array}$ & $\begin{array}{l}\text { Dialeptic } \rightarrow \text { Automotor(Bil. diffuse } \\
\text { Hand) }\end{array}$ & Rt.RSC & Rt.HH,MTG \\
\hline 11 & Bil.TPO & fear and déjà vu & $\begin{array}{l}\text { Automotor(olimentary and Bil.TPO } \\
\text { Bil.Hand) }\end{array}$ & Lt.RSC,Lt.Prc & Lt.HH,PHG,ITS \\
\hline 12 & Bil.TPO & - & $\begin{array}{l}\text { Automotor(olimentary and Bil.TPO } \\
\text { Lt.Hand) }\end{array}$ & Lt.RSC,lingual gyrus & Lt.Prc,Rt.RSC,Prc \\
\hline
\end{tabular}

\section{Preoperative findings}

The mean age at epilepsy onset was 11 years (min-max:1-18), and the mean epilepsy duration was 10 years (min-max:1-27).Four patients had normal MRI results before surgery.Preoperative MRI showed a left-sided thalamic infarction in one patient(pt.9).MRI of two patients (pts.7 and 10)showed anterior prelobectomy anterior temporal lesions Three patients (pts.3,4 and 9)showed posterior cingulatelesions on MRI. Theremaining two showed parieto-occipital lesions. PET and MEG were almost diffuse.

\section{Semiological characteristics}

\section{Aura}

Six of twelve included patients experienced auras, one patient experienced vestibular responses (pt.3), and three patients (pts. 1,pt. 4 and pt. 10) experienced dizziness and visual aura(i.e.,blurred visionin both eyes).Patient 10 experienced complex auditory aura and fear, while one patient experienced fear and déjà $\mathrm{vu}(\mathrm{pt.7)}$.

\section{Seizure semiology}

Five of the 12 included patients experienced complex motor seizures (pts. 8, 9, 10, 11 and 12), three experienced dialeptic seizures (pts. 1, 2 and 3),and four others experienced automotor seizures (pts.4,5,6 and 7).Patients9,10,11 and 12moved as if they were looking for something followed by alternating 
movements of the eyes/head in different directions. Patient 8 exhibited trunk and hip movement accompanied by flapping motions in the bilateral upper limb extremities.

\section{Interictal and ictal EEG data}

All patients underwent video-EEG monitoring. Interictal epileptiform discharges occurred ipsilateral to the side of the EZ in four patients (pts.5, 8, 9 and 10). Discharges were non-lateralized in six patients, and two patients exhibited no interictal discharge(pts.3and11).In seven patients, interictal EEG signals were mainly localized to the temporo-parieto-occipital area. In the remaining five patients, interictal discharges wereanterior-middle temporal in two cases and non-localized in the other patients.

In seven patients (pts.1,2,6,7,8,11 and 12), the site of seizure onset was primarily localized in the temporal-parietal-occipital area, but the ictal onset occurred ipsilateral to the $E Z$ in only two of seven cases, and the others were non-lateralized. In the remaining patients, ictal EEG signals were in the temporal area (pts.4 and 9)or generalized.

\section{Anatomical-electrical-clinical correlations based on SEEG findings}

With respect to ictal semiology, three of twelve patients showed consciousness impairments (pts.1,2 and3). The ictal SEEG trace of patient 2 revealed that seizure onset was characterized by the appearance of polyspike-slowwave discharge in the posteriorcingulate cortex (mesial contacts of electrodes D1-3,V14,and T1-4) and precuneus(mesial contacts of electrodes S1-3).Meanwhile, déjà vuaura appeared. Then, seizure activity spread to the inferior parietal lobule (lateral contacts of electrodesS11-12,D12-13, and V10-11).At this time, dialeptic seizures were observed(Fig. 1).

Four patients (pts. 4, 5,6 and 7) had bilateral hand fumbling. The ictal SEEG recording of patient 7 revealed that seizure onset occurred in the PCC (mesial contacts of electrodes T2-4). After $600-800$ milliseconds, ictal activity spread to the $\mathrm{Pcu}(\mathrm{S} 5-6)$ and long insular gyrus(V2-3), and vestibular aura was observed (Fig. 2).

Five of twelve patients were characterized by complex motor seizures with ictal spread to the PCC, MCC,cuneus, mesial temporal structures or long insular gyrus of the ipsilateral hemisphere. The ictal SEEG trace of patient 8 revealed that seizure onset was characterized by the appearance of ripple discharges (medial contacts of electrodesS1-3,V2-3,and D1-2) and then spread to the Pcu(medial contacts of electrodesW2-3) and MCC(medial contacts of electrodes R1-3 andM1-3)in the absence of clinical treatment. After $5 \mathrm{~s}$, the seizure activity had spread to the hippocampus (medial contacts of electrodes B2-3), lingual gyrus and para-hippocampus (medial contacts of electrodes E2-3and Z2-3), and superior temporal gyrus (V12-13). He attempted to get up from the bed,which was followed by alternating movements of the eyes/head in different directions as if he was looking for something(Fig. 3).

However, for two patients (pts.3 and 4)with posterior cingulatelesions, the seizure onset zone was the contralateral hippocampus. The ictal SEEG recording of patient 4 revealed that seizure onset was 
characterized by the appearance of polyspike-slowwave discharge in the left hippocampus head(mesial contacts of electrodesB1-3).Approximately 600 milliseconds later, seizure activity spread to the left isthmus(mesial contacts of electrodesX2-3) and right hippocampus head (mesial contacts of electrodesB'1-3).One second later, dialeptic and automotor activity appeared once the seizure had spread to the right PCC lesion( $\mathrm{R}^{\prime} 2-3$ andS'2-3), precuneus(mesial contacts of electrodes T'2-3) and IPL (lateral contacts of electrodesC'12-13,S'10-13,and V'13-14)(Fig. 4).

\section{Surgery,epileptic outcomes and pathology}

Seven patients underwent posterior cingulate gyrus resection following SEEG evaluation. Theywere seizure free after 24 months of follow-up (Engle I).In this subset of patients, the mean follow-up after resection was 42 months (range 34-64 months). Pathologic abnormalities included type 1 focal cortical dysplasia (5 patients), FCD IIIB(pt.4) and cerebral injury (pt.5).

Five patients underwent SEEG-guided radiofrequency thermocoagulation; three patients remained seizure free after 12 months of follow-up, but the remaining patients (pt. 1 and pt.3) had $50 \%$ fewer seizures (Engel III)(Table 1).

However, for two patients with posterior cingulatelesions, the seizure onset zone was the contralateral hippocampus. Patient 4, who underwent posterior cingulate gyrus resection, had seizure freedom after 24 months of follow-up. Patient 3, whounderwent SEEG-guided radiofrequency thermocoagulation, had a $50 \%$ reduction in seizures.

\section{Discussion}

Posterior cingulate epilepsy is a rare and diagnostically challenging form of epilepsy owing to the particular anatomical position of the posterior cingulate [9]. In the present study, we analysed semiological characteristics and EEG/SEEG data to identify anatomic-electrical-clinical relationships in 12 patients with posterior cingulate epilepsy.

Previous studies have revealed that posterior cingulate epilepsy is associated with several types of premonitory auras[10].In one such study, dyscognitive aura(déjà vu, jamais vuor depersonalization), abdominal aura,gustatory aura, and sensations of falling or movement were reported in three patients. Two patients had multipleauras. The dyscognitive aura and abdominal aura were elicited by stimulation of the limbic structures of the temporal lobe[2].Our findings are mostly consistent with these previous studies, although visual and vestibular aura occurred more frequently in our patient group, which was elicited by electrical stimulation of the humanposterior cingulate cortex[11].

It was difficult to localize the ictal onset areas based on scalp EEG.Previous reports have shown that scalp interictal and ictal EEG lateralize or localize the seizures correctly in $50 \%-60 \%$ of posterior cingulate epilepsy cases[1]. In the present study, we found that interictal or ictal epileptiform discharges were most observed in the temporo-parieto-occipital region but little in the temporal region, which was different from that observed in TLE. Interictal and ictal discharges in temporal areas were detected in the scalp EEG in 
only three patients (pts.2,4 and 10). However, two patients (pts.2 and 10), whose seizures resembled temporal lobeseizures $[4,9]$, underwent anterior temporal lobectomy but failed to achieve a seizure-free outcome.We used SEEG to reevaluate patients and found that the seizure onset zone (SOZ) was localized in the posterior cingulate gyrus. Two patients who had undergone posterior cingulate gyrus surgery were seizure-free at the end of the follow-up period.

Therefore, it is difficult to distinguish PCG from non-PCE via scalp EEG, particularly the mesial-temporal lobe epilepsy.SEEG represents a unique method for identifying and characterizing the underlying physiological mechanisms of PCE.

In SEEG methodology, the EZ is defined as the site of origin for epileptic seizures[12].Therefore, we focused our SEEG analyses mainly on the identification of the brain regions in which specific ictal patterns developed during seizures. The regions associated with the primary organization of ictal discharges and subsequent propagation were correlated with the anatomical positions of the electrodes that displayed the maximal abnormal activity and with the evolution of clinical seizure signs.

We analysed a case series of twelve patients in the present study, seven of whom exhibited temporal lobe seizures $[1,2,4]$, and the remaining patients had complex motor behaviours. Our study revealed that the semiology of PCE can involve dialeptic or automotor seizures, which depended upon the seizure spreading to mesialtemporal structures, the precuneus[13]or the IPL.Structural connections via cingulate tracts were found between the IPL and PCC in all subjects with both streamline and probabilistic analyses $[14,15]$. These findings are consistent with the previous hypothesisthat alterationsin consciousness and automatisms in PCE reflect the involvement of the temporal lobes $[1,4,9]$.

Complex motor behaviours may also be observed in the latecourse of posterior cortex seizures, once the seizure propagates to more anterior regions[1].In the present study,the most common clinical characteristics of complex motor behaviours were exploratory gaze movements and bilateral hand fumbling movements. In these patients, complex motor behaviours were observed only when ictal activity assessed by SEEG had spread to the precuneus, MCC, medial temporal lobe and superior temporal gyrus $[12,16]$.The cingulum bundle was predominantly composed of cingulate fibres that head in either a rostral or caudal direction, with the majority bifurcating to go in both directions. The PCG interlinks medial parts of the frontal, parietal, and temporal lobes by the cingulum bundle[17]. The highest number of active sites was found in the ventral and dorsal aMCC, whose stimulation triggered a variety of goal-oriented behavioursinvolvingreaching and grasping actions and exploratory gaze movements[11].Therefore, the clinical characteristics of posterior cingulate epilepsy vary among patients that are different from the mesial-temporal lobe epilepsy[18].

This preliminary impression could suggest a higher specificity of the SEEG method in mapping the EZ[5].Among this group, seven of twelve patients who underwent PCG resection were seizurefree at the end of the follow-up period. The three of five patients who underwent RF-TC achieved seizurefreedom, and the remaining patients had $30 \%-50 \%$ fewer seizures. SEEG methodology is both safe and effective in patients with difficult-to-localize, medically intractable, focal epilepsies[5].RF-TC selectively disrupts 
critical hubs in the epileptic network through contiguous contacts within the range of a single electrode and provides a more curative effect[19]in regions such as the small and deeply epileptogenic foci,PCG.

Accurate description of the brain regions involved in seizure genesis is a crucial objective in the context of epilepsy surgery. However,the identification of the EZ is based on the abilities of experienced clinical neurophysiologists to identify the relevant anatomic-electrical-clinical correlations and SEEG patterns.In the present study, two patients (pts.3 and 4) had posterior cingulate lesions identified by MRI but had contralateral hippocampal onset identified by SEEG in our study.One patient(pt.4) who underwentposterior cingulate lesion resection was seizurefree.After treating the posterior cingulate lesion using SEEG-guided RT-FC, the discharge of the hippocampus was obviously reduced(pt.3). On the one hand,postoperative pathology was FCDIIIb(FCD Icand ganglion glioma), which is often associated with malformations during cortical development, in particular focal cortical dysplasia (FCD), which is most often the basis of epilepsy lesions. Invasive EEG investigations may provide useful information, although in GNT-associated focal epilepsy, the main goal of intracerebral recordings is usually to map the eloquent cortex in the proximity of the neoplasm[20].

On the other hand,the posterior cingulate cortex is known to project to the hippocampus via the entorhinal and parahippocampal cortex and to receive direct and indirect connections from the hippocampus. The inferior cingulum bundle is a white matter tract projecting from the PCC to the hippocampus or parahippocampus and the entorhinal cortex[21,22]. Ictal propagation to the cingulate gyrus has frequently been observed among patients with temporallobe epilepsy[18].However,YCShih found in patients with left mesial temporal lobe epilepsy with hippocampal sclerosis, the left inferior cingulum bundle underwent degeneration in tandem with the left hippocampus volume, whereas intrinsic functional connectivity seems to react by compensating for the loss of connectivity. Their results suggested that increased intrinsic functional connectivity of the contralesional hippocampus was a compensatory response to decreased hippocampal connectivity on the lesion side[23].According to the hypothesis, we speculate that the PCG lesion and hippocampus functional connectivity decreased,but the contralesional PCG and hippocampus have extensive unctional connections. Fundamental to the original concept of the EZ was the idea of one or more regions of brain involved in the primary organization of the ictal discharge, rather than a "focus". Indeed, from the outset of SEEG development, the EZ was seen as a set of interconnected regional systems[24]. The now widely accepted notion of epileptogenic networks stems from these early observations and is intrinsically related to the SEEG method of recording.The EZ in the PCG may be an epileptogenic network that is distributed across the limbic system and is complex[25].

This study has some limitations.First, this study was definitely limited by the location or number of implanted electrodes designed to treat these epilepsy patients. This spatial limitation in the recordings may have caused difficulties in identifying the wholeseizure pathway.Second, the sample size in this study was relatively small.Further studies employing a large number of participants would be helpful to confirm these preliminary results. We should apply CCEP or other methods to precisely track the pathway through which the seizures spread. 


\section{Conclusions}

The clinical characteristics of PCE are complex and varied. Aura type (e.g., blurred vision in both eyes and vestibular responses), dialeptic seizures, and complex motor seizures are the main indicators of PCE.The classifications of scalp EEG were regional parieto-occipital and posterior temporal,but little lateralization. Analysis of high-quality SEEG data can help to identify the relevant anatomic-electrical-clinical correlations. Our findings indicate that the clinical characteristics of PCE are different among patientsand that the final electroclinical phenotype depends on the pattern of seizure spread.For patients with pharmacoresistant posterior cingulated epilepsy,favourable outcomes (Engel class I) could be observed by using SEEG. For patients with posterior cingulate epilepsy, the SEEG findings suggest that the seizure onset zone and epileptogenic zone can be two different areas.

\section{Abbreviations}

3D: Three-dimensional; CT: Computed tomography; EEG: Electroencephalography; EZ: Epileptogenic zone;SOZ:seizure onset zone; MCC: Middle cingulate cortex; MRI: Magnetic resonance imaging; PCC: Posterior cingulate cortex; PCL: Paracentral lobule; PET: Positron emission tomography; MCG:Magnetoencephalography;PoCG: Postcentral gyrus; PrC: Precuneus; PrCG: Precentral gyrus; SEEG: Stereoeletroencephalography; SMA: Supplementary motor area; IPL: Inferior parietal lobule;FCD:Focal cortical dysplasia; RT-FC: Radiofrequency thermocoagulation;CCEP: cortico-cortical evoked potential

\section{Declarations}

\section{Ethics approval and consent to participate}

The study was performed under a protocol approved by the ethic committee of Sanbo Hospital of Capital Medical University, Beijing, China.

The ethic committee of the hospital granted me permission to access the medical records for the purpose of this study. The study participants and their caregivers agreed to participate in this study, and all provided written informed consent. The consent obtained covered the publication of personal and potentially identifying information such as age and gender.

\section{Consent for publication}

Informed consents to publish were obtained from the patients with individual data involved in this article. 


\section{Availability of data and materials}

The datasets during and analyzed during the current study are available

from the first and corresponding authors on reasonable request.

\section{Competing interests}

None of the authors has any conflict of interest to disclose. We confirm that

we have read the Journal's position on issues involved in ethical publication

and affirm that this report is consistent with those guidelines.

\section{Funding}

This work supported by National Natural Science Foundation of China

(81671285).

\section{Authors' contributions}

ZY contributed to the data acquisition and analysis of the manuscript. WJ

and QD contributed to the data acquisition.JZ and GGanalysis and redaction of the

manuscript, and also the interpretation of the data. YJ,FZand XF contributed

to the data acquisition. MW and GL contributed to redaction of the manuscript. All

authors read and approved the final manuscript.

\section{Acknowledgements}

We would like to thank all the patients who participated in this study.

\section{References}

1. Garzon E, Lüders HO. Cingulate epilepsy. In: Lüders HO, editor. Textbook of epilepsysurgery. London: Informa Healthcare; 2008. pp. 334-53.

2. Powell R, Elwes R, Hamandi K, Mullatti N. Cingulate gyrus epilepsy. Pract Neurol. 2018;18:447-54.

3. Koubeissi MZ, Jouny CC, Blakeley JO, Bergey GK. Analysis of dynamics and propagation of parietal cingulate seizures with secondary mesial temporal involvement. Epilepsy Behav. 2009;14:108-12.

4. Enatsu R, Bulacio J, Nair DR, Bingaman W, Najm I, Gonzalez-Martinez J. Posterior cingulate epilepsy: clinical and neurophysiological analysis. J Neurol Neurosurg Psychiatry. 2014;85:44-50. 
5. Gonzalez-Martinez J, Bulacio J, Alexopoulos A, Jehi L, Bingaman W, Najm I.

Stereoelectroencephalography in the "difficult to localize" refractory focal epilepsy: early experience from a North American epilepsy center. Epilepsia. 2013;54:323-30.

6. Luders H, Acharya J, Baumgartner C, Benbadis S, Bleasel A, Burgess R, et al. Semiological seizure classification. Epilepsia. 1998;39:1006-13.

7. Kahane P, Landre E, Minotti L, Francione S, Ryvlin P. The Bancaud and Talairach view on the epileptogenic zone: a working hypothesis. Epileptic Disord. 2006;8(Suppl 2):16-26.

8. Engel JJ, Van Ness P, Rasmussen TB, Ojemann LM. Outcome with respect to epileptic seizures. In: Engel J, editor. Surgical treatment of the epilepsies. New York: Raven Press; 1993. pp. 609-21.

9. Dalmagro CL, Bianchin MM, Velasco TR, Alexandre V, Walz R, Terra-Bustamante VC, et al. Clinical features of patients with posterior cortex epilepsies and predictors of surgical outcome. Epilepsia. 2005;46:1442-9.

10. Alkawadri R, So NK, Van Ness PC, Alexopoulos AV. Cingulate epilepsy: report of 3 electroclinical subtypes with surgical outcomes. JAMA Neurol. 2013;70:995-1002.

11. Caruana F, Gerbella M, Avanzini P, Gozzo F, Pelliccia V, Mai R, et al. Motor and emotional behaviours elicited by electrical stimulation of the human cingulate cortex. Brain. 2018;141:3035-51.

12. Munari C, Bancaud J. The role of stereo-electro-encephalography (SEEG) inthe evaluation of partial epileptic patients. In: Porter RJ, Morselli PL, editors. The epilepsies. London: Butterworths; 1987. pp. 267-306.

13. Yang $Y$, Wang $H$, Zhou W, Qian $T$, Sun W, Zhao G. Electroclinical characteristics of seizures arising from the precuneus based on stereoelectroencephalography (SEEG). BMC Neurol. 2018;18:110.

14. Vogt BA, Laureys S. Posterior cingulate, precuneal and retrosplenial cortices: cytology and components of the neural network correlates of consciousness. Prog Brain Res. 2005;150:205-17.

15. Khalsa S, Mayhew SD, Chechlacz M, Bagary M, Bagshaw AP. The structural and functional connectivity of the posterior cingulate cortex: comparison between deterministic and probabilistic tractography for the investigation of structure-function relationships. Neuroimage. 2014;102 Pt 1:118 $-27$.

16. Vogt BA. Midcingulate cortex: structure, connections, homologies, functions and diseases. J Chem Neuroanat. 2016;74:28-46.

17. Bubb EJ, Metzler-Baddeley C, Aggleton JP. The cingulum bundle: anatomy, function, and dysfunction. Neurosci Biobehav Rev. 2018;92:104-27.

18. Dolezalova I, Brazdil M, Kahane P. Temporal lobe epilepsy? Things are not always what they seem. Epileptic Disord. 2017;19:59-66.

19. Wang D, Wei P, Shan Y, Ren L, Wang Y, Zhao G. Optimized stereoelectroencephalography-guided radiofrequency thermocoagulation in the treatment of patients with focal epilepsy. Ann Transl Med. 2020;8:15. 
20. Giulioni M, Marucci G, Martinoni M, Marliani AF, Toni F, Bartiromo F, et al. Epilepsy associated tumors: review article. World J Clin Cases. 2014;2:623-41.

21. Pearson JM, Heilbronner SR, Barack DL, Hayden BY, Platt ML. Posterior cingulate cortex: adapting behavior to a changing world. Trends Cogn Sci. 2011;15:143-51.

22. Aminoff EM, Kveraga K, Bar M. The role of the parahippocampal cortex in cognition. Trends Cogn Sci. 2013;17:379-90.

23. Shih YC, Tseng CE, Lin FH, Liou HH, Tseng WY. Hippocampal atrophy is associated with altered hippocampus-posterior cingulate cortex connectivity in mesial temporal lobe epilepsy with hippocampal sclerosis. AJNR Am J Neuroradiol. 2017;38:626-32.

24. Bartolomei F, Nica A, Valenti-Hirsch MP, Adam C, Denuelle M. Interpretation of SEEG recordings. Neurophysiol Clin. 2018;48:53-7.

25. Bartolomei F, Lagarde S, Wendling F, McGonigal A, Jirsa V, Guye M, et al. Defining epileptogenic networks: contribution of SEEG and signal analysis. Epilepsia. 2017;58:1131-47.

\section{Figures}




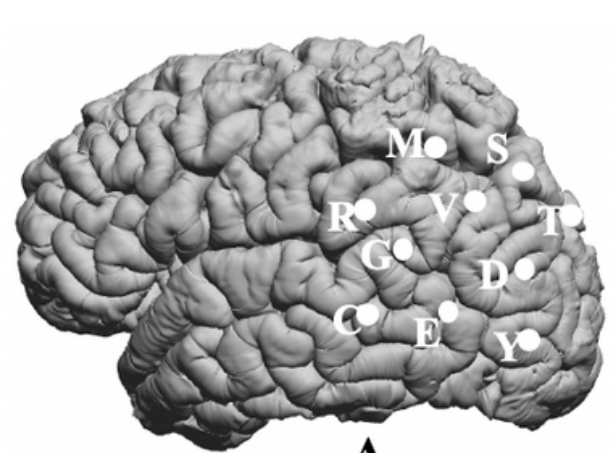

A

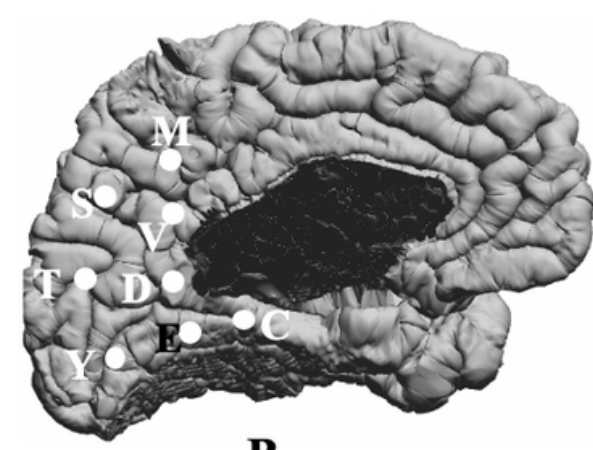

B

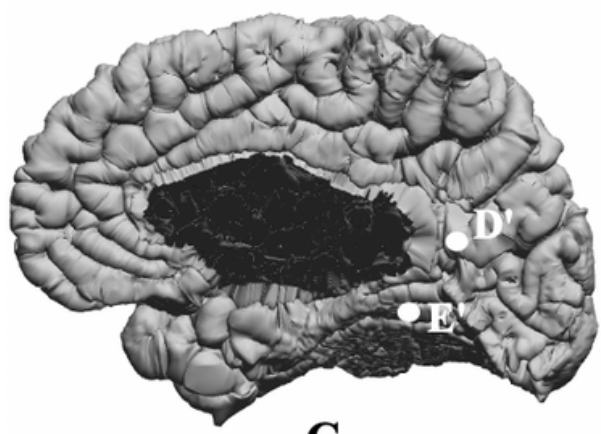

C

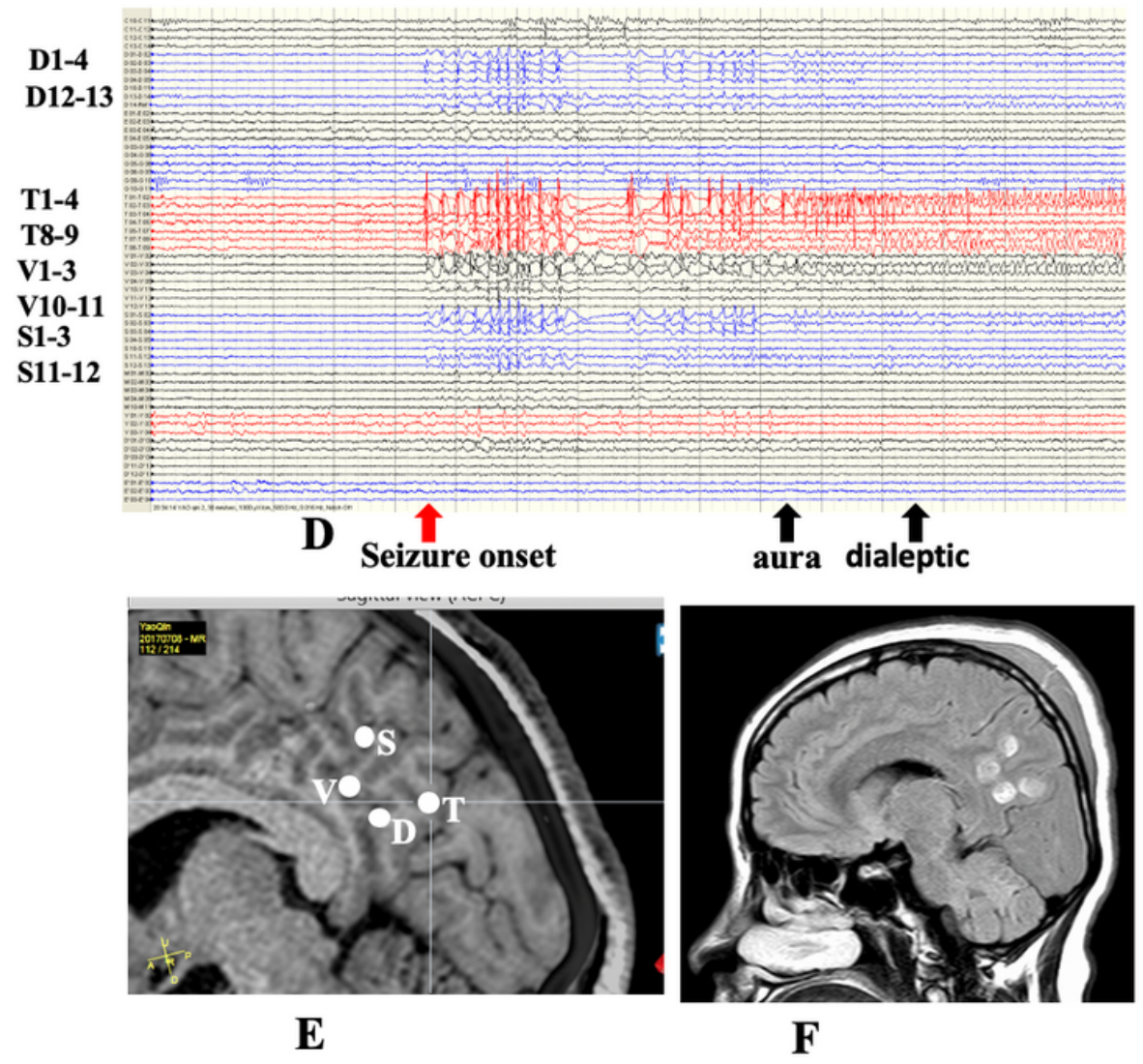

Figure 1

A-B-C:SEEG electrode implantation on lateral and medial view of 3D brain of Patient (A-Left lateral, B-Left medial;C-Right medial); D:Ictal SEEG traces show that the seizure onset was record from PCG(red arrow) (medial contacts of electrode T1-4, S1-3,D1-4 and V1-3) with déjà vu being observed. After 3 seconds, the ictal activity spread to T8-9,S11-12,D12-13, the clinical sign of dialeptic seizure appeared. E:Ictal onset electrode"T,D,V,S" located left PCG. F:post-RFTC sagittal MRI after RF-TC treatment 


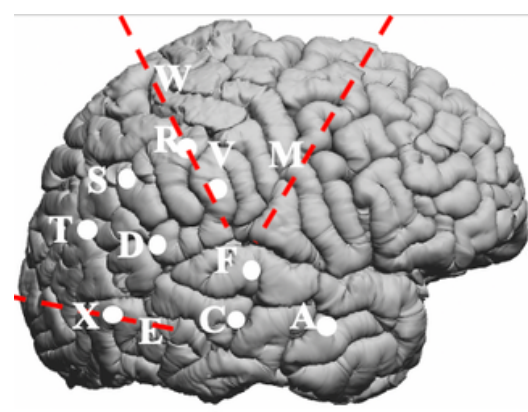

A

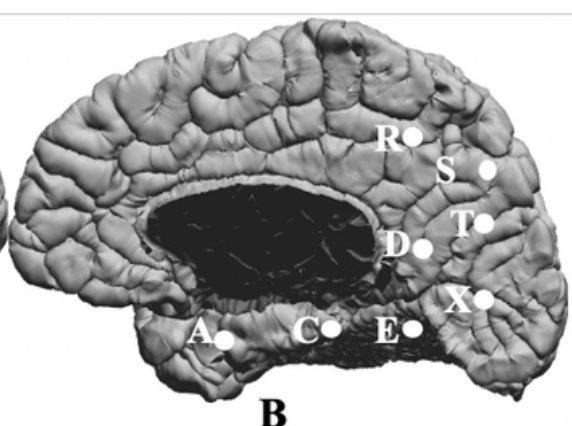

B
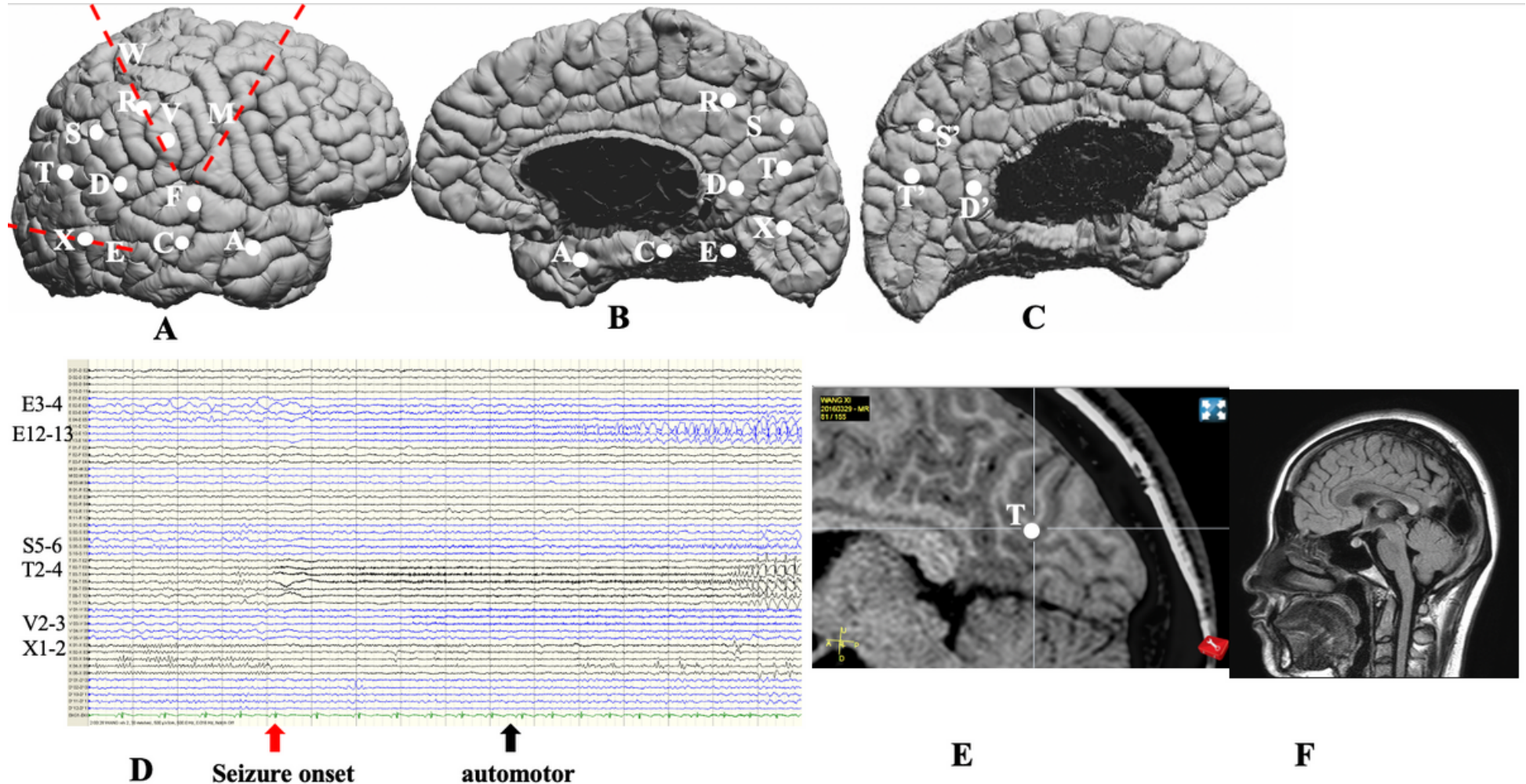

Figure 2

A-B-C:SEEG electrode implantation on lateral and medial view of 3D brain of Patient (A-Left lateral, B-Left medial;C-Right medial); D:Ictal SEEG traces show that the seizure onset was record from PCG(red arrow) (medial contacts of electrode T2-4) with no clinical sign being observed. When the ictal activity spreads to Pcu (medial contacts of electrode S5-6), long insular gyrus (medial contacts of electrode V2-3), ITG (lateral contacts of electrode E12-13), PHG(medial contacts of electrode E3-4 ),Cu(medial contacts of electrode X1-2), the semiology of automotor seizure appeared.凶E:Ictal onset electrode"T"located left PCG. $\mathrm{F}:$ Postsurgery MRI:resection of the posterior cingulate gyrus and precuneus. 


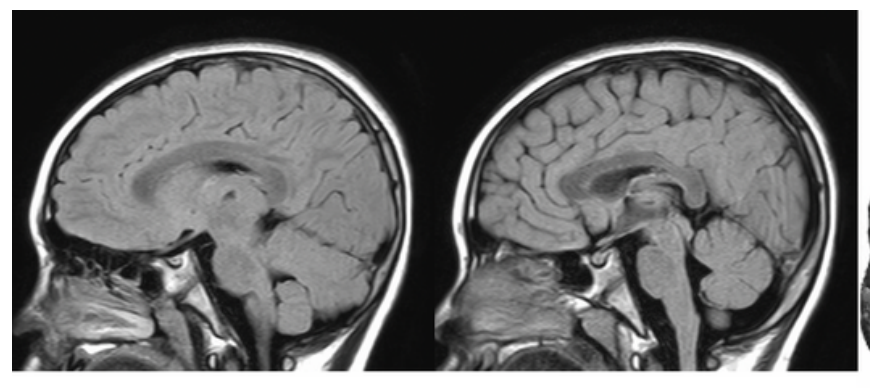

A
B

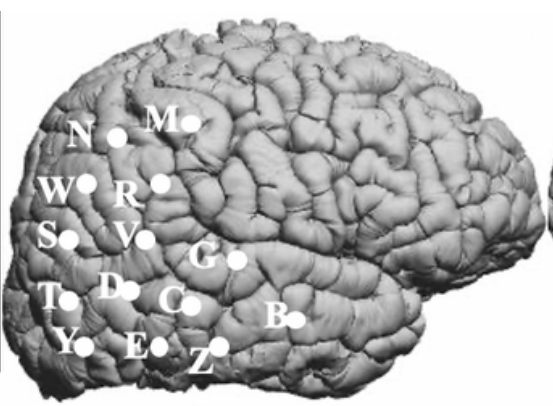

C

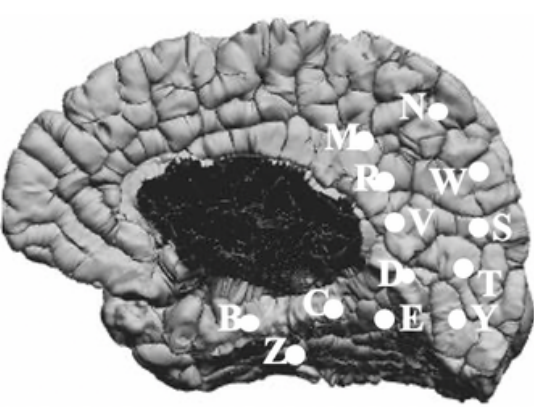

D

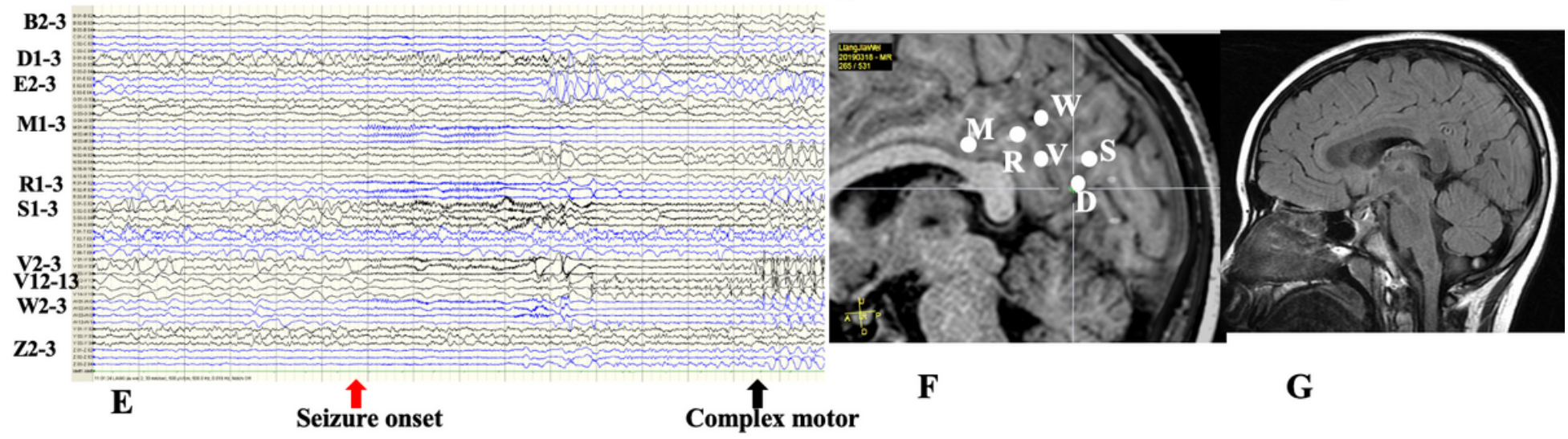

Figure 3

A-B:Brain MRI of presurgery; C-D:SEEG electrode implantation on lateral and medial view 3D brain of Patient $\triangle \mathrm{E}$ :Ictal SEEG traces show that the seizure onset was record from PCG(red arrow) (medial contacts of electrode S1-3,V2-3,R1-3),RSC(medial contacts of electrode D1-3) and MCC(medial contacts of electrode M1-3) with no clinical sign being observed. After 2 second, the ictal activity spread to $\mathrm{HH}$ (medial contacts of electrode B2-3),PHG (medial contacts of electrode E2-3 and Z2-3),STG(lateral contacts of electrode V12-13). The clinical sign of complex motor seizure appeared after 12 seconds . F:Ictal onset electrode"S,D,V,R,W" located left PCG. F:post-RFTC sagittal MRI after RF-TC treatment 


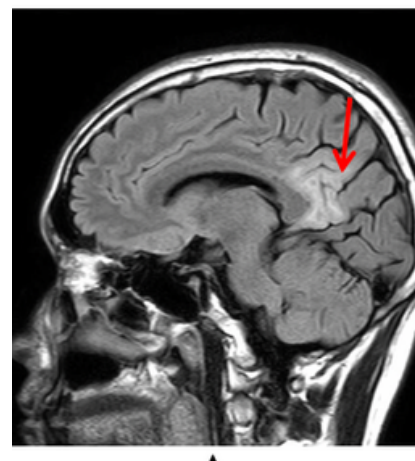

$\mathbf{A}$
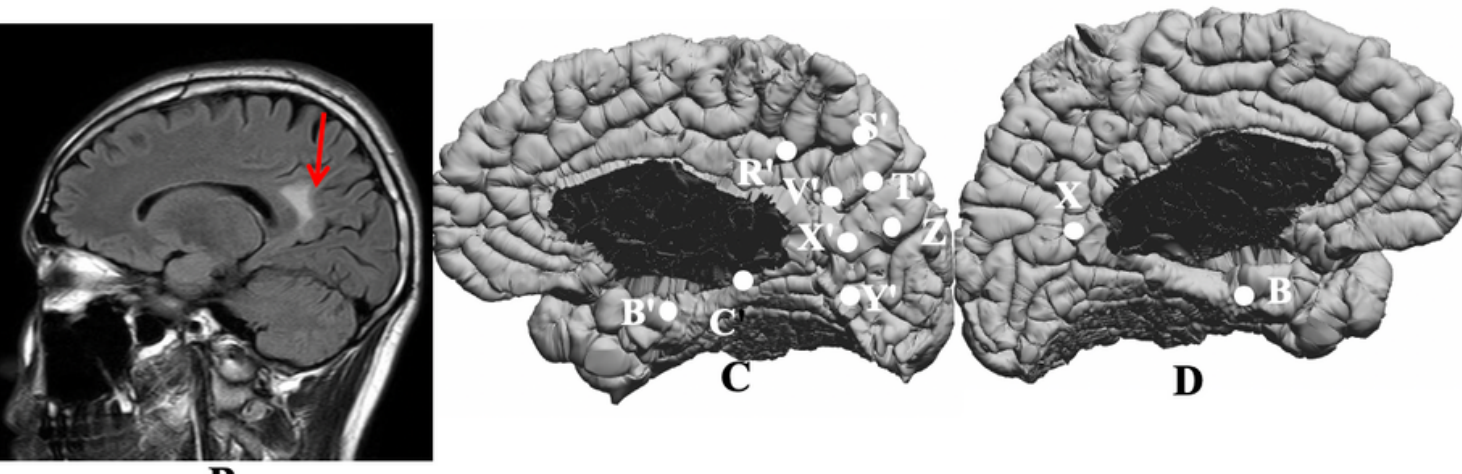

B

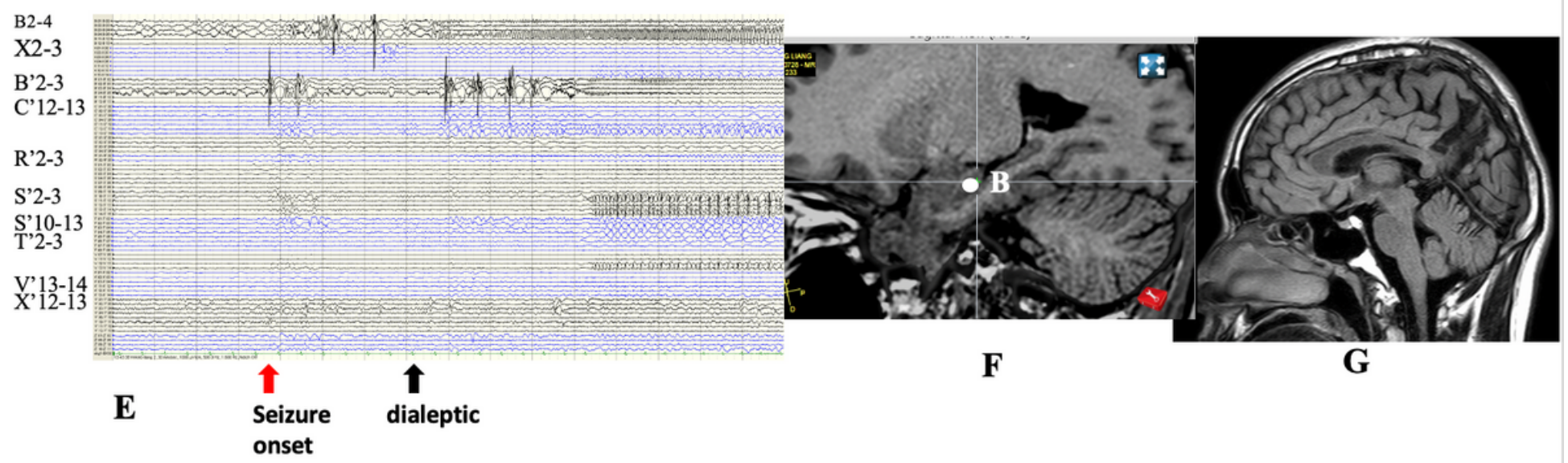

Figure 4

A-B:Brain MRI of presurgery;C-D:SEEG electrode implantation on right and left Hemisphere medial view of three-dimensional (3D) brain of Patient\E:Ictal SEEG traces show that the seizure onset was record from hippocampcus ( $\mathrm{HH}$,red arrow)(medial contacts of electrode B2-4) with no clinical sign being observed. When the ictal activity spread to X2-3, theX2-3,B'2-3, R'2-3,S'2-3, the clinical sign of dialeptic seizure appeared .F:ictal onset electrode"B" located left HH.G:postsurgery MRI:resection of the posterior cingulate gyrus and precuneus. 